Elsevier required licence: (C) <2017>. This manuscript version is made available under the CC-BY-NC-ND 4.0 license http://creativecommons.org/licenses/by-nc-nd/4.0/ 


\section{Special Issue on Localization and Mapping in Challenging Environments}

Reliable localization and mapping are competencies essential to realize many different robot applications. A broad range of techniques, ranging from statistical approaches such as Kalman filters and particle filters, to optimization based methods, have been developed in the last two decades for solving these problems. Significant contributions have been made in improving the efficiency and robustness of robot localization and mapping in both 2D and 3D scenarios using different sensors such as laser scanners, monocular cameras, RGB-D sensors and many others. However, it is still a significant challenge to achieve robust localization and mapping in environments populated with dynamic or deformable objects, underground with uneven terrain, underwater in turbid conditions, and in environments with changing lighting conditions. This special issue aims to consolidate some recent developments in localization and mapping in challenging environments, providing state-of-the art approaches and algorithms that are able to facilitate the use of mobile robots in a range of application domains.

Twenty-nine papers were received out of which eighteen were accepted after a few rounds of reviews and revisions. Accepted papers cover a large range of topics including localization, tracking and mapping in dynamic environments with laser sensors, localization using acoustic sensors, Simultaneous Localization and Mapping (SLAM) in underwater environments, 3D point cloud registration and 3D reconstruction, RGB-D SLAM in dynamic environments, global localization, loop closure detection, and industry applications of robot localization and mapping.

Four papers discuss robust vehicle navigation in outdoor environments with a laser scanner as the primary sensor. Wei et al. propose a strategy to detect moving/dynamic vehicles by using the difference between two consecutive scans and a likelihood-field-based vehicle measurement model. Registration is achieved by the combination of coarse motion estimation and fine batch adjustment. Wang et al. study the pedestrian recognition and tracking problem for autonomous vehicles using a 3D LiDAR. A classifier trained by a support vector machine is used to recognize pedestrians, so that a timely prediction of pedestrian motions can be provided to the autonomous vehicle platform. In the paper by Li et al., a Gaussian Mixture Model (GMM) is proposed to represent a point cloud acquired from a 3D sensor. A hierarchical structure is employed to deal with the problem of the density variations at different ranges thereby increasing the robustness of the scan registration. Furthermore, when the platform is moving while scanning, a probabilistic graph is constructed by assigning each Gaussian component with a pose. Droeschel et al. propose a SLAM method where laser-range measurements are aggregated by registering sparse 3D scans with a local multi-resolution surfel map that has high resolution in the vicinity of the robot and coarser resolutions with increasing distance. A globally consistent dense 3D map of the environment is built through graph optimization and registration of incrementally built local dense 3D maps of nearby key poses. The drivability of the terrain is assessed by analyzing the height differences in an allocentric height map. The system has been successfully used in the DARPA Robotics Challenge and the DLR SpaceBot Camp.

Laser scanners are widely used for mapping in many applications. However, glass, mirrors, shiny or translucent surfaces cause erroneous measurements depending on the incident angle of the laser beam. Two papers in this special issue have addressed this challenge. Wang and Wang propose a simple and effective solution to identify glass panels by detecting the reflected light intensity profile around the normal incident angle to the glass panel. The glass detection method is integrated with SLAM algorithm and is shown to be able to detect and localise glass obstacles in real-time with around $95 \%$ accuracy. Koch et al. deal with the problem of mapping transparent and specular reflective objects with an extended version of the Mirror Detector Approach, named Reflection Classifier Approach. The characteristics identified in the measurements of different materials such as shiny metals, mirrors, and glasses, from different distances and angles are investigated. Experiments show that discrimination of transparent and reflective materials based on the reflected intensity is 
possible and feasible.

Two papers discuss point cloud registration and SLAM using RGBD sensors for indoor environments. Serafin and Grisetti use a novel strategy to search for correspondences, resulting in a point cloud registration method that can operate on-line and cope with measurements gathered from a heterogeneous set of range and depth sensors. An efficient map-merging procedure is used to quickly update the tracked scene and handle dynamic aspects. Sun et al. propose a novel RGB-D data based motion removal approach and integrated it into the front end of RGB-D SLAM. The motion removal approach acts as a pre-processing stage to filter out data that were associated with moving objects, making the RGB-D SLAM effective in challenging dynamic environments.

There are three papers on important industry applications of SLAM. Ozog et al. consider the mapping of underwater ship hulls using vision. They proposed a model-assisted bundle adjustment framework in which visually-derived features are fused with an underlying 3D mesh provided a priori, for example, from CAD drawings. Quintana et al. present a method for automatic 3D scanning of furnished buildings using a mobile robot equipped with a 3D laser scanner. The robot autonomously navigates in cluttered indoor environments where the next location for the robot to move is calculated using a probabilistic Next Best Scan algorithm. Comparisons to related methods and evaluations in real and simulated environments are presented. Beinschob et al. present an approach to Automated Guided Vehicle (AGV) deployment which aims to drastically reduce the time and cost of the installation. A SICK 3D laser scanning technology is employed to build an accurate and consistent virtual representation of the whole warehouse environment. By utilizing segmentation and processing, a semantic map is generated, in a semi-automated manner. This map contains 3D geo-referenced positions as well as a $2 \mathrm{D}$ localization map.

Two papers address the localization and mapping problem using unconventional low quality sensors. Dirafzoon et al. present an approach for global exploration and mapping of unknown environments using a swarm of cyborg insects, known as biobots, for emergency response scenarios under minimal sensing and localization constraints. Binary local interactions among the biobots when they are within a set distance of each other is the only piece of information provided. The biobots and an aerial leader are used to explore and map a region of interest. Local maps are constructed using coordinate free encounter information of the agents. These maps are then merged into a global map which can be visualized using a graphical representation. Zhong et al. propose sound source localization methods based on robot head motion and interaural time difference (ITD) data. This study presents two robotic hearing approaches based on a human perception model that combines ITD and head turn motion data to locate sound sources. The first method is based on recognizing the changing trends of the cross-correlation function of binaural inputs. The second method uses an extended Kalman filter that estimates the orientation of a sound source by fusing the robot's self-motion and ITD data to reduce the localization errors recursively. This method can function with limited memory resources and is able to keep tracking the relative position change of a number of static sources when the robot moves.

Four papers are related to visual navigation and visual SLAM. The paper by Pire et al. describes a real-time feature-based stereo SLAM system that is robust and accurate in a wide variety of conditions such as indoors/outdoors environments with dynamic objects and changing light conditions. The system follows the parallel-tracking-and-mapping (PTAM) strategy which includes a tracking thread that estimating the camera pose at frame rate; and a mapping thread that updates a keyframe-based map at a lower frequency. Ji et al. consider the automatic calibration method for camera sensor networks based on 3D texture map information. Using a novel image descriptor based on Quantized Line parameters in the Hough space for 2D-3D matching, the 6DOF camera parameters are estimated by applying a particle filter-based optimization scheme. Yang et al. present a visual SLAM system that integrates measurements from two cameras on board a micro aerial vehicle (MAV). The two cameras do not need to have overlapping fields of view. The resulting visual 
SLAM system enables autonomous navigation of an MAV in complex scenarios. Latif et al. propose a novel loop closure detection algorithm for visual navigation based on solving a sparse optimization problem. The formulation requires no offline dictionary learning and thus allows online incremental operation.

Biswas and Veloso introduce the Episodic non-Markov Localization (EnML), a novel robot localization algorithm in changing environments. EnML reasons about observations arising from permanent, temporary, or moving objects. The maximum likelihood estimate of the belief is incrementally computed by non-linear functional optimization. EnML limits the history of observations and pose estimates to "episodes" over which the belief is computed. Different types of sensors including laser rangefinders and depth cameras could be used in EnML. EnML is demonstrated using a team of indoor autonomous service mobile robots traversing hundreds of kilometers.

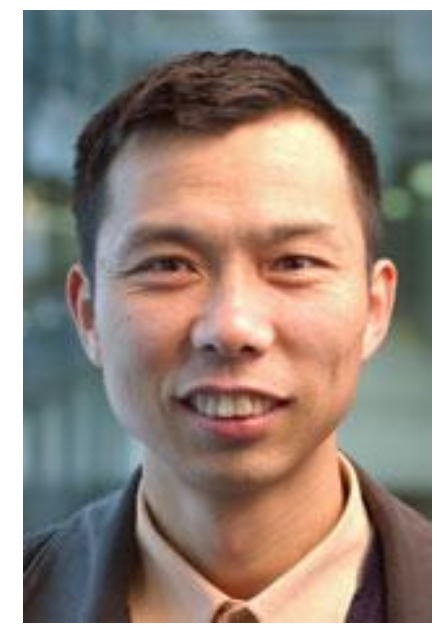

Shoudong Huang received the Bachelor and Master degrees in Mathematics, Ph.D in Automatic Control from Northeastern University, P.R. China in 1987, 1990, and 1998, respectively. He is currently an Associate Professor at Centre for Autonomous Systems, Faculty of Engineering and Information Technology, University of Technology, Sydney, Australia. His research interests include nonlinear control systems and mobile robots simultaneous localization and mapping (SLAM), exploration and navigation.

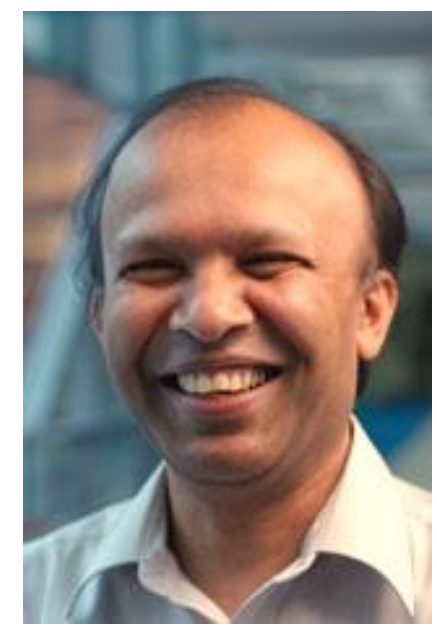


Gamini Dissanayake is the James N Kirby Professor of Mechanical and Mechatronic Engineering at University of Technology, Sydney (UTS). His current research interests are in the areas of localization and map building for mobile robots, navigation systems, dynamics and control of mechanical systems, cargo handling, optimization and path planning. He leads the Centre for Autonomous Systems at UTS. He graduated in Mechanical/Production Engineering from the University of Peradeniya, Sri Lanka. He received his M.Sc. in Machine Tool Technology and Ph.D. in Mechanical Engineering (Robotics) from the University of Birmingham, England in 1981 and 1985, respectively.

\section{Guest Editors}

Shoudong Huang

Centre for Autonomous Systems, Faculty of Engineering and IT

University of Technology Sydney, PO Box 123, Broadway, NSW 2007, Australia

Email address: shoudong.huang@uts.edu.au

\section{Gamini Dissanayake}

Centre for Autonomous Systems, Faculty of Engineering and IT

University of Technology Sydney, PO Box 123, Broadway, NSW 2007, Australia

Email address: gamini.dissanayake@uts.edu.au 